\title{
Transfer of small negative atmospheric ions
}

$\operatorname{AUTHOR}(\mathrm{S})$ :

Maruyama, T; Katayama, T

\section{CITATION:}

Maruyama, T ...[et al]. Transfer of small negative atmospheric ions. JOURNAL OF APPLIED PHYSICS 2003, 94(11): 7365-7369

\section{ISSUE DATE:}

2003-12-01

URL:

http://hdl.handle.net/2433/43539

\section{RIGHT:}

Copyright 2003 American Institute of Physics. This article may be downloaded for personal use only. Any other use requires prior permission of the author and the American Institute of Physics. 


\title{
Transfer of small negative atmospheric ions
}

\author{
Toshiro Maruyama ${ }^{\text {a) }}$ and Takashi Katayama \\ Department of Chemical Engineering, Graduate School of Engineering, Kyoto University, \\ Kyoto 606-8501, Japan
}

(Received 18 March 2003; accepted 20 September 2003)

\begin{abstract}
The transfer of small negative atmospheric ions in air and from air to water has been studied. The mean lifetime of ions was experimentally obtained from the concentration distribution of ions in a round free jet. It was about $30 \mathrm{~s}$ in relative humidity of $63 \%-75 \%$, indicating that ions generated at one source in a room could not be homogeneously spread throughout the room by conventional convective flow of air. Rapid dissipation of the ions occurs during diffusion in stationary air. However, most of the ions that enter the mouth of a human body reach the lungs, because of the high convective velocity of breathing. The irradiation of ions onto water decreased the electric conductivity of the water. This finding suggests that the transfer of ions into water in a human body breaks the network of $\mathrm{H}_{2} \mathrm{O}$ molecules and enhances the formation of hydrogen bonds between a biopolymer and water. (C) 2003 American Institute of Physics. [DOI: 10.1063/1.1625438]
\end{abstract}

\section{INTRODUCTION}

Small atmospheric ions are approximately 1-nm-diam particles which possess either a positive or negative unit electric charge. These small ions are formed by the ionization of air molecules due to natural mechanisms, e.g., cosmic rays, ground radioactivity, and radioactive decay of radon, thoron, and their derivatives which are given off by the ground and mix with the atmosphere. Negative ions are generated from neutral molecules or atoms attached by an electron. The most prevalent negative ion is $\mathrm{O}_{2}^{-}$because $\mathrm{O}_{2}$ has the highest electron affinity $(0.15 \mathrm{eV})$ among the molecules in air. Finally, $\mathrm{O}_{2}^{-}\left(\mathrm{H}_{2} \mathrm{O}\right)_{n}, \mathrm{CO}_{4}^{-}\left(\mathrm{H}_{2} \mathrm{O}\right)_{n}$, and $\mathrm{NO}_{3}^{-}\left(\mathrm{H}_{2} \mathrm{O}\right)_{n}$ are formed in pure atmosphere $\left(\mathrm{N}_{2}, \mathrm{O}_{2}, \mathrm{CO}_{2}\right.$, and $\left.\mathrm{H}_{2} \mathrm{O}\right)$. Among them, the dominant negative small ion is $\mathrm{O}_{2}^{-}\left(\mathrm{H}_{2} \mathrm{O}\right)_{n}$, while the oxonium ion $\mathrm{H}_{3} \mathrm{O}^{+}\left(\mathrm{H}_{2} \mathrm{O}\right)_{k}$ is the dominant positive small ion. ${ }^{1,2}$ The concentration of small ions in air is normally $300-500$ ions per $\mathrm{cm}^{3}$ at ground level; this value may be reduced to less than $10 \%$ in a very polluted atmosphere or in clouds.

Small negative atmospheric ions have attracted attention because the application of ions to the human body shows prominent effects on physiological reactions. Recently, various kinds of artificial generators of small atmospheric negative ions have been used to compensate for the decrease of natural ions in a house. The ions generated are transported to the human body by diffusion and convection in air. Efficient transport is crucial, because the adhesion of ions to particles (aerosol) and combination with water molecules change the small ions into large ions (charged aerosol) which do not affect the human body. The mean time interval between the appearance of a small ion and its attachment to another particle is relatively short.

This article deals with diffusive and convective transfer of small negative ions in air. An analytical solution will be

\footnotetext{
a) Author to whom correspondence should be addressed; electronic mail: maruyama@cheme.kyoto-u.ac.jp
}

given for determining the diffusion of ions in stationary air. We discuss the measured mean lifetime of an ion convectively transferred by a free round jet. A simple model is used to calculate the convective transport of ions from the human mouth to the lungs during breathing. The transfer of negative ions from air to water is also discussed.

\section{MEAN LIFETIME OF SMALL NEGATIVE IONS IN AIR}

The temporal variations of the concentration $n$ of small ions in air are expressed as

$$
\begin{aligned}
& d n_{+} / d t=q-\alpha n_{+} n_{-}-\eta_{+0} n_{+} N_{0}-\eta_{+-} n_{+} N_{-}, \\
& d n_{-} / d t=q-\alpha n_{+} n_{-}-\eta_{-0} n_{-} N_{0}-\eta_{-+} n_{-} N_{+},
\end{aligned}
$$

where $N$ represents the concentration of large particles. Subscripts,+- , and 0 refer to positive, negative, and neutral electric charges, respectively. $\alpha$ represents the coefficient of recombination of small ions with ions of opposite sign, and $\eta$ is the coefficient of adhesion of small ions to particles (aerosol). The first term on the right-hand side, $q$, expresses the generation rate of an ion pair, the second term dissipation by the recombination of small ions, and the third and fourth terms dissipation of the small ions upon adhesion to large neutral and opposite-sign particles, respectively.

If we assume that $n_{+}=n_{-}$and that the mobility of a negative ion is equal to that of a positive ion, Eqs. (1) and (2) can be approximated by

$$
d n / d t=q-\alpha n^{2}-2 \eta_{+-} n_{+} N_{-}=q-\alpha n^{2}-\beta n N .
$$

An estimate en $^{3,4}$ for application to the atmosphere on land shows that $n=10^{8} \mathrm{~m}^{-3}, N=10^{10} \mathrm{~m}^{-3}$, and $\alpha$ and $\beta$ $=10^{-12} \mathrm{~m}^{3} \mathrm{~s}^{-1}$. Therefore, Eq. (3) can be further approximated by

$$
d n / d t=q-\beta n N .
$$

When generation is stopped, i.e., $q=0$, the concentration of ions after $t \mathrm{~s}$ is expressed as 


$$
n=n_{0} \exp (-\beta N t) .
$$

The mean lifetime of ions $\tau$ is defined as

$$
\tau=1 /(\beta N) .
$$

Bricard and Pradel $^{5}$ described the value of $\tau$ as $30-50 \mathrm{~s}$ under normal conditions, but their method of determining this was not clarified.

\section{A. Transfer of small negative ions by a free round jet}

A Gerdien condenser was used to measure the concentration of externally generated ions in air. In this meter, the flow of air is created by a fan to introduce ions into the meter. Therefore, this meter cannot be used to measure the concentration of ions in stationary air, i.e., the diffusion of ions. This meter should be used to measure the ion concentration in convective flow, where the flow rate is large enough to minimize the effect of the air flow of the meter. In this study transfer by a free round jet was selected to measure the mean lifetime of small negative ions in air.

The basic equation of convection with dissipation by collision with large particles is expressed as

$$
U_{j} \partial n / \partial r+V_{j} \partial n / \partial z=-\beta n N,
$$

where $U_{j}$ and $V_{j}$ represent the radial and axial component of the velocity of the jet and $z$ the axial distance. Multiplication by $2 \pi r$ and an integration from $r=0$ to $r \rightarrow \infty$ yield

$$
\begin{aligned}
& \int_{0}^{\infty} 2 \pi r U_{j}(\partial n / \partial r) d r_{+} \int_{0}^{\infty} 2 \pi r V_{j}(\partial n / \partial z) d r \\
& \quad=-\beta N \int_{0}^{\infty} 2 \pi r n d r .
\end{aligned}
$$

For simplicity in calculation, the velocity $V_{j}$ and concentration $n$ in the cross section of the jet are approximated as constant, that is, it is assumed that $V_{j}=V_{j}(z)$ and $n=n(z)$. By applying the equation of continuity the first term of the left-hand side of Eq. (8) can be expressed as $n\left(\partial Q_{\mathrm{j}} / \partial z\right)$ and consequently, Eq. (8) is expressed in simple form as

$$
d\left(Q_{j} n\right) / d z=-\beta n N A_{j},
$$

where $Q_{j}$ represents the volumetric flow rate of the jet and $A_{j}$ is the cross-sectional area of the jet. The mean lifetime $\tau$ can be obtained from

$$
\tau=1 / \beta N=-\left(A_{j} n\right) /\left[d\left(Q_{j} n\right) / d z\right] .
$$

For changes of the radius and mean velocity of the jet with $z$, the law of the conservation of momentum is assumed:

$$
Q_{j} V_{j}=Q_{j 0} V_{j 0} \text {. }
$$

The diameter ${ }^{6}$ of the jet at $z$ is given by

$$
d_{j}=d_{j 0}+2 z \tan (\theta / 2),
$$

where $\theta=14^{\circ}$.

\section{B. Experiment}

The mean lifetime of small negative ions in air was measured from the concentration distribution along a free round jet. A dc corona discharge was used as the ion generator. A

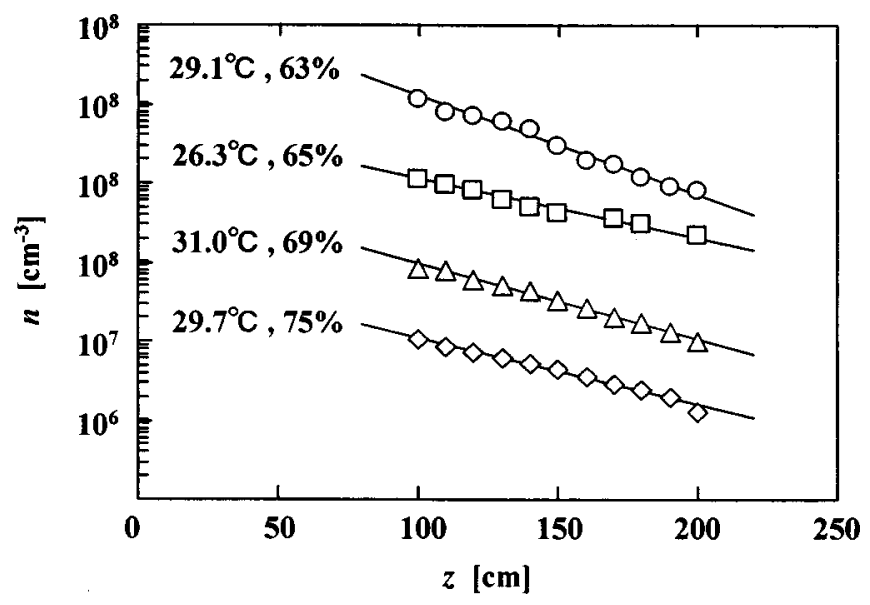

FIG. 1. Concentration of small negative atmospheric ions along the axis of the round free jet for various relative humidities of air.

fan was attached to the generator to transport ions by a free round jet. A dc voltage of $-9 \mathrm{kV}$ was applied to a tapered single electrode of the ion generator. The small negative ions generated were conveyed by air flow from a hole $3 \mathrm{~cm}$ in diameter at a flow rate of $1.6 \times 10^{4} \mathrm{~cm}^{3} \mathrm{~min}^{-1}$. The air flow formed a free round jet in a wide-open space. The concentration of small negative ions along the axis of the jet was measured using a Gerdien-type air ion counter (Universal, IC-1000), which introduces air flow at a rate of 4.8 $\times 10^{4} \mathrm{~cm}^{3} \mathrm{~min}^{-1}$. The measuring distance ranged from 100 to $200 \mathrm{~cm}$, where the flow rates of the jet, $1.47 \times 10^{5}$ and $2.78 \times 10^{5} \mathrm{~cm}^{3} \mathrm{~min}^{-1}$, are higher than that of the ion counter.

\section{Results and discussion}

Figure 1 shows the concentration $n$ of small negative ions in air as a function of the axial distance $z$ of the round free jet for various relative humidities of air. Apparently, the concentration decreases exponentially with an increase in $z$. Substituting the initial conditions of the jet, diameter $d_{j 0}$ $=3 \mathrm{~cm}$ and flow rate $Q_{j 0}=1.6 \times 10^{4} \mathrm{~cm}^{3} \mathrm{~min}^{-1}$, in Eqs. (11) and (12), and then substituting these equations and the measured concentration of small negative ions into Eq. (10) yield the mean lifetime of the ions. Figure 2 shows the obtained mean lifetime $\tau$ as a function of axial distance $z$ of the free round jet. The rather large variations in the results are attrib-

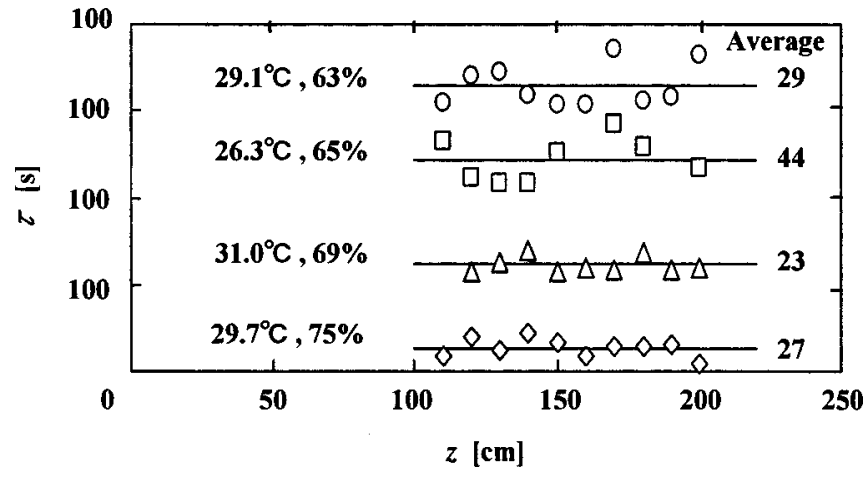

FIG. 2. Lifetime of small negative atmospheric ions along the axis of the round free jet for various relative humidities of air. 


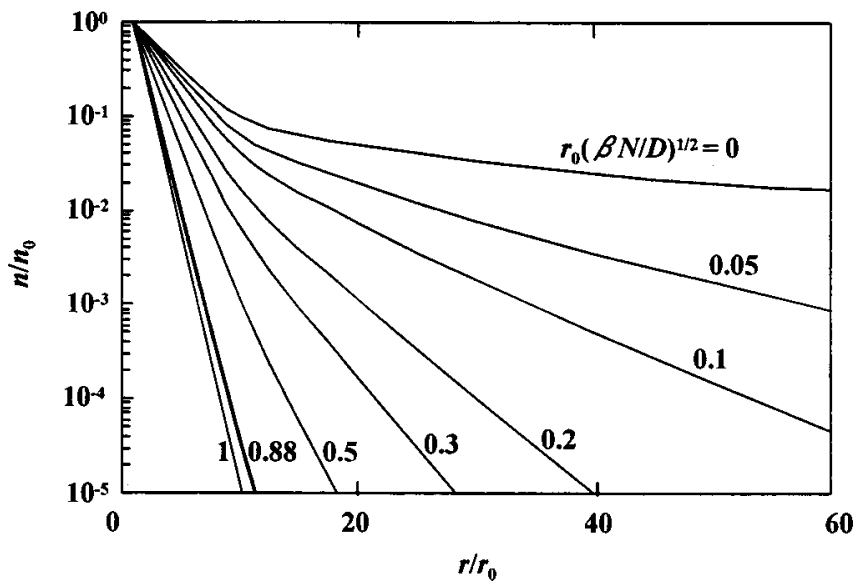

FIG. 3. Normalized concentration $n / n_{0}$ of small negative atmospheric ions as a function of $r_{0}(\beta N / D)^{1 / 2}$ and $r / r_{0}$.

utable to differentiation of nonsmoothing data in the calculations. The average values of the mean lifetime are also shown in Fig. 2. Although the deviation is rather large, there seems to be no dependence on the relative humidity. The mean lifetime is about $30 \mathrm{~s}$ for relative humidity of $63 \%-$ $75 \%$. In general, the circulation time of air flow forced by a fan or an air conditioner in a room is several minutes. Thus the mean lifetime is short compared to the circulation time of forced air flow in a room. This finding indicates that small negative ions generated at one source in a room cannot be homogeneously spread throughout the room by conventional convective air flow, and consequently a room cannot be treated as a concentrated system when applying Eqs. (1)-(6).

\section{DIFFUSION OF SMALL NEGATIVE IONS IN STATIONARY AIR}

Under most conditions where carrier diffusion in gases is considered, the electrostatic forces between individual carriers can be neglected. ${ }^{7}$ The basic equation of diffusion with dissipation by collision with large particles is expressed as

$$
\left(1 / r^{2}\right) d\left(r^{2} D d n / d r\right) / d r=\beta n N,
$$

where $D$ represents the diffusion coefficient of the ion, and $r$ the radial distance from the source. The boundary conditions are

$$
\begin{aligned}
& n=n_{0} \quad \text { at } r=r_{0}, \\
& n=0 \quad \text { at } r=\infty .
\end{aligned}
$$

The solution is expressed with two dimensionless combined variables, $r_{0}(\beta N / D)^{1 / 2}$ and $r / r_{0}$, as

$$
n / n_{0}=\exp \left[-r_{0}(\beta N / D)^{1 / 2}\left(r / r_{0}-1\right)\right] /\left(r / r_{0}\right) .
$$

Figure 3 shows the normalized concentration $n / n_{0}$ of the small negative atmospheric ions as a function of $r_{0}(\beta N / D)^{1 / 2}$ and $r / r_{0}$. The curve for $r_{0}(\beta N / D)^{1 / 2}=0$ indicates diffusion of an ion with infinitely long mean lifetime. Figure 3 shows the decrease in concentration with the progression of diffusion. The decrease is accelerated by a decrease in mean lifetime.

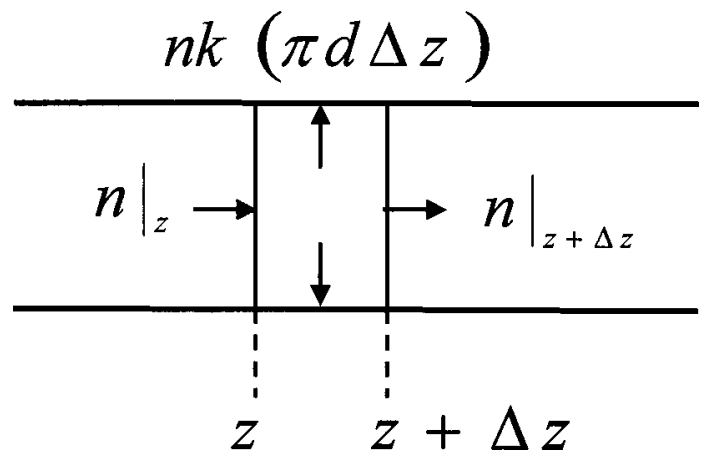

FIG. 4. Shell balance at $\Delta z$ of trachea.

In 1899, Townsend evaluated the values of the coefficient of diffusion $D$ to be 0.028 and $0.043 \mathrm{~cm}^{2} \mathrm{~s}^{-1}$ for positive and negative ions in air, respectively. ${ }^{7}$ Substituting both the diffusion coefficient of $0.043 \mathrm{~cm}^{2} \mathrm{~s}^{-1}$ and the mean lifetime of $30 \mathrm{~s}$ for negative ions in air yields $r_{0}(\beta N / D)^{1 / 2}$ $=0.88$ for $r_{0}=1 \mathrm{~cm}$. The curve for $r_{0}(\beta N / D)^{1 / 2}=0.88$ in Fig. 3 indicates that the decrease in concentration is marked. That is, $n / n_{0}$ is $3.62 \times 10^{-5}$ at $r=10 \mathrm{~cm}$. Therefore, negative ions should be transferred to a remote location by convective flow of air.

\section{CONVECTIVE TRANSFER OF SMALL NEGATIVE IONS FROM MOUTH TO LUNGS BY BREATHING}

A simple pipe-flow model is used to calculate the convective transport of ions in a human body from the mouth to the lungs by breathing. The air flow during inspiration through the trachea from the mouth to the lungs was approximated as flow at constant velocity through a straight circular pipe with a rigid wall. For simplicity in calculation, the concentration distribution across the trachea is approximated as flat and the transfer of ions to the trachea wall by radial diffusion is expressed using the mass transfer coefficient. Thus, the basic equation of convective transport with dissipation by collisions with large particles and the trachea wall is obtained via a shell balance, as shown in Fig. 4.

$$
W \Delta n=-n k_{\mathrm{av}}(\pi d \Delta z)-\left(\pi d^{2} / 4\right) \Delta z \beta n N .
$$

Here, $W, k_{\mathrm{av}}, d$, and $z$ represent the volumetric flow rate of air, space-averaged mass-transfer coefficient of the ion, the diameter of the trachea, and the axial distance. The limit $\Delta z \rightarrow 0$ yields the differential equation,

$$
V d n / d z=-\left(4 k_{\mathrm{av}} / d+\beta N\right) n,
$$

where $V$ represents the velocity of air.

The boundary condition is

$$
n=n_{0} \quad \text { at } z=z_{0} .
$$

The solution is expressed as

$$
n / n_{0}=\exp \left[-\left(4 k_{\mathrm{av}} / d+\beta N\right) z / V\right] .
$$

Table I lists the characteristics of human breathing ${ }^{8}$ and air. Under the assumption that the period of inspiration is equal to that of expiration, the time-averaged volumetric flow rate was calculated by multiplying the air volume inspired by twice the frequency of breathing. The space-averaged mass- 
TABLE I. Characteristics of breathing and air.

\begin{tabular}{ll}
\hline \hline Representative diameter of trachea, $d$ & $2.5 \mathrm{~cm}$ \\
Length of trachea from mouth to lungs, $L$ & $25 \mathrm{~cm}$ \\
Volume of inspiration & $300 \mathrm{~cm}^{3}$ \\
Frequency of breathing & $18 \mathrm{~min}^{-1}$ \\
& \\
Time-averaged volumetric flow rate of inspiration & $180 \mathrm{~cm}^{3} \mathrm{~s}^{-1}$ \\
Time-averaged velocity of inspiration & $36.7 \mathrm{~cm} \mathrm{~s}^{-1}$ \\
Density of air (at $\left.20^{\circ} \mathrm{C}\right)$ & $1.286 \times 10^{3} \mathrm{~g} \mathrm{~cm}^{-3}$ \\
Viscosity of air $\left(\right.$ at $\left.20^{\circ} \mathrm{C}\right)$ & $1.80 \times 10^{4} \mathrm{~g} \mathrm{~cm}^{-1} \mathrm{~s}^{-1}$ \\
$\left(\operatorname{Re}=655 ; S c=3.26 ; S h_{\mathrm{av}}=9.69\right)$ & \\
\hline
\end{tabular}

transfer coefficient $k_{\mathrm{av}}$ was obtained from the correlation for the space-averaged Sherwood number $S h_{\mathrm{av}}$ in a laminar pipe flow,

$$
S h_{\mathrm{av}} \equiv k_{\mathrm{av}} d / D=1.62(\operatorname{Re} S c d / L)^{1 / 3} .
$$

Substituting these values into Eq. (17) yields $n / n_{0}=0.815$, which indicates that most of the small negative atmospheric ions entering from the mouth reach the lungs. This is attributable to the high convective velocity, which overcomes the rapid dissipation that occurs in stationary air.

\section{TRANSFER OF SMALL IONS FROM AIR TO WATER}

For the ion to be effective for physiological reactions in the human body, some characteristics of the ions must be transferred to the solid and liquid matter which constitute a human body. In particular, not only transfer of the charge of ions but also some changes in the structure of liquid molecules are expected to occur during the transfer of ions from air to liquid. Here in Sec. V, changes in the structure of water molecules during such transfer is discussed on the basis of experimental results obtained by bombarding small atmospheric ions onto the surface of water.

\section{A. Experiment}

The surface of water in a $100 \mathrm{~cm}^{3}$ beaker $(63 \mathrm{~mm}$ in inner diameter and $86 \mathrm{~mm}$ in depth) was bombarded by negative and positive ions generated by the ion generator (Eco Holistic). Dc voltages of $-6,-9$, and $+9 \mathrm{kV}$ were applied to the tapered single electrode of the ion generator. The electric conductivity of water was measured using a conductivity meter (M\&S Instruments, CD-35MII). The cell of the conductivity meter was completely immersed in the water.

In a preliminary study, the viscosity, surface tension, $p \mathrm{H}$, permittivity, and electric conductivity of water were measured in a nonirradiated state after being irradiated for several days. It was confirmed that irradiation did not cause any irreversible changes in these physical properties.

\section{B. Results and discussion}

Figure 5 shows the electric conductivity of water as a function of the distance $z$ from the tip of the electrode to the surface of the water. The irradiation of positive ions has no effect on the electric conductivity. However, the irradiation of negative ions decreases the electric conductivity. The difference from that of the nonirradiated state increases with a

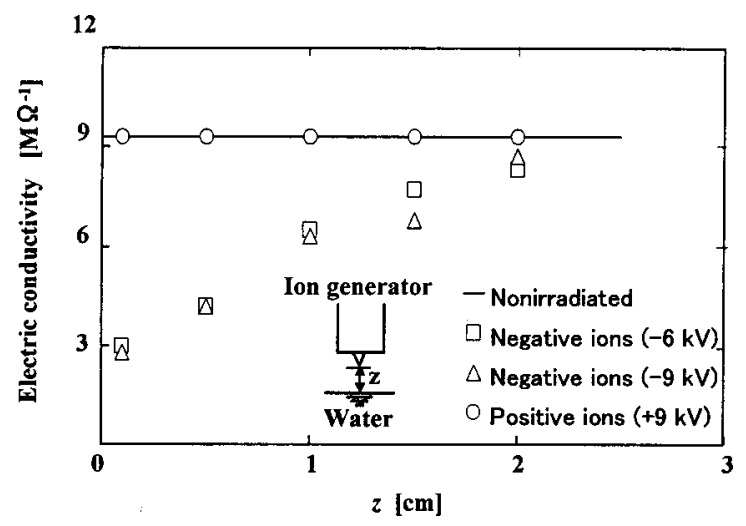

FIG. 5. Electric conductivity of water as a function of the distance $z$ from the tip of the electrode to the surface of the water.

decrease in distance $z$ at voltages of -6 and $-9 \mathrm{kV}$. These findings suggest that the hydrogen bond of water is broken by the irradiation of negative ions. The mechanism can be explained as follows. The positive ion is the oxonium ion $\mathrm{H}_{3} \mathrm{O}^{+}\left(\mathrm{H}_{2} \mathrm{O}\right)_{k}$ which smoothly transmits its charge to the network of $\mathrm{H}_{2} \mathrm{O}$ via the proton-exchange reaction. ${ }^{9-11}$ However, the dominant negative ion $\mathrm{O}_{2}^{-}\left(\mathrm{H}_{2} \mathrm{O}\right)_{n}$ does not smoothly transfer its charge to the network of $\mathrm{H}_{2} \mathrm{O}$. Furthermore, its electric field destroys the network of $\mathrm{H}_{2} \mathrm{O}$ by attracting $\mathrm{H}_{2} \mathrm{O}$ molecules as a different ion species in water. The disproportionation of the superoxide anion radicals $\mathrm{O}_{2}^{-}$in water yeilds $\mathrm{H}_{2} \mathrm{O}_{2}$ and $\mathrm{O}_{2}$ if there is no substrate. ${ }^{12}$ The fact that any irreversible change in the physical properties was not detected after irradiation suggests that the accumulation of $\mathrm{H}_{2} \mathrm{O}_{2}$ in water is very small.

The hydrogen bond between a biopolymer and water plays an essential role in the function of the biopolymer through acceleration of the dissolution, formation of the three-dimensional structure, and assurance of the stability. The destruction of the network of $\mathrm{H}_{2} \mathrm{O}$ by the irradiation of negative ions enhances the formation of the hydrogen bond between water and the biopolymer. Thus the irradiation of negative ions onto a human body contributes to physiological reactions.

\section{CONCLUSIONS}

The mean lifetime of small negative atmospheric ions is about $30 \mathrm{~s}$ in relative humidity of $63 \%-75 \%$. This is too short a time for the ions generated at one source in a room to be spread homogeneously throughout the room by conventional convective air flow. Rapid dissipation of the ions occurs during diffusion in stationary air. However, most of the ions entering the mouth of a human body reach the lungs, because of the high convective velocity of breathing.

The irradiation of ions onto water decreases the electric conductivity of water. This finding suggests that the transfer of ions into water in a human body breaks the network of $\mathrm{H}_{2} \mathrm{O}$ and enhances the formation of hydrogen bonds between a biopolymer and water. 


\section{ACKNOWLEDGMENT}

The authors thank Y. Komaki of Eco Holistic Inc. for providing the ion generator.

${ }^{1}$ M. L. Huertas and J. Fontan, Atmos. Environ. 9, 1018 (1975).

${ }^{2}$ M. L. Huertas, J. Fontan, and J. Gonzalez, Atmos. Environ. 12, 2351 (1978).

${ }^{3}$ H. Israël, Atmospharische Elektrizität I. Akademische Verlagsgesellshaft (Geest-Portig K.-G., Liepzig, 1957).

${ }^{4}$ J. A. Chalmer, Atmospheric Electricity, 2nd ed. (Pergamon, Oxford, 1967).

${ }^{5}$ J. Bricard and J. Pradel, in Aerosol Science, edited by C. N. Davies (Academic, London, 1966), p. 87.
${ }^{6}$ L. L. Simpson, in Turbulence in Mixing Operation, edited by R. S. Brodkey (Academic, New York, 1975), p. 277.

${ }^{7}$ L. B. Loeb, Basic Processes of Gaseous Electronics (University of California Press, Berkeley, 1960).

${ }^{8}$ J. K. Inglis, A Textbook of Human Biology, 3rd ed. (Butterworth, Heinemann, 1988).

${ }^{9}$ M. Eigen and L. De Maeyer, Proc. R. Soc. London, Ser. A 247, 505 (1958)

${ }^{10}$ S. Meiboom, J. Chem. Phys. 34, 375 (1961).

${ }^{11}$ M. Eigen, Angew. Chem., Int. Ed. Engl. 3, 1 (1964).

${ }^{12}$ J. M. McCord and I. Fridovich, J. Biol. Chem. 243, 5753 (1968). 